\title{
Melanoma associated with congenital intermediate common blue nevus of the scalp - Case report ${ }^{*}$
}

\author{
Camila Sampaio Ribeiro ${ }^{1}$ \\ Maria Auxiliadora Jeunon Sousa ${ }^{3}$
}

\author{
Sergio S. Serpa ${ }^{2}$ \\ Thiago Jeunon ${ }^{1}$
}

DOI: http:/ / dx.doi.org/10.1590/abd1806-4841.20164248

\begin{abstract}
Melanomas can arise either de novo (70\%) or from pre-existing melanocytic lesions (30\%). Of the latter, most cases arise at the dermoepidermal junction from small congenital or acquired non-blue nevi while only a few arise from blue nevi, notably the cellular subtype and less commonly the common (dendritic) type. Melanomas that arise from blue nevi usually occur on the scalp with greater frequency, as in the case described. Although previous studies have discussed melanoma arising from giant congenital blue nevi, few have discussed those arising from intermediate blue nevi. We present a case of a 52-yearold man with melanoma on the scalp evolving from an intermediate congenital common blue nevus.
\end{abstract}

Keywords: Blue nevus; Congenital abnormalities; Melanoma; Scalp

\section{INTRODUCTION}

Congenital melanocytic nevus (CMN) is defined, clinically, as a melanocytic lesion present at birth or which develops during infancy from preexistent melanocytes. ${ }^{1-3}$ Histologically, it is characterized by the presence of melanocytes between collagen bundles at the reticular dermis and around adnexal structures.

Classification of $\mathrm{CMN}$, regarding its dimensions, may take in consideration its area, the body area percentage that it occupies or only its largest diameter. In the latter situation, congenital nevi are defined as small (with less than $1.5 \mathrm{~cm}$ in diameter), medium/ big (with $1.5 \mathrm{~cm}$ to $19.9 \mathrm{~cm}$ in diameter) and giant (with $20 \mathrm{~cm}$ or more in diameter). ${ }^{1.5}$

The majority of congenital nevi are less than $4 \mathrm{~mm}$ in diameter. On the other hand, giant nevi occur in 1:20,000 to 1:50,000 newborns. ${ }^{1,4}$
Histopatologically, melanocytic nevi are classified as blue and non blue. In non blue nevi, the formation of groups of melanocytes (nests and/or strands) is observed. According to the distribution of these nests in the skin layers, nevi may be classified as junctional, compound or intradermal. On the other hand, blue nevus (BN) shows no tendency to groupings, but rather a diffuse proliferation of dermal melanocytes and no formation of nests. ${ }^{4}$

The risk of developing melanoma over a $\mathrm{CMN}$ is a very well established subject. It is believed that, this risk is directly proportional to nevus size, varying from $2.6 \%$ to $4.9 \%$ for small and medi- um nevi and from $6 \%$ to $20 \%$ for giant nevi. ${ }^{1,6}$ Considering the overall frequency of melanomas, around $30 \%$ arise on a preexistent nevi, while $70 \%$ are de novo melanomas. ${ }^{7}$

We present a case of melanoma arising within a congenital intermediate $\mathrm{BN}$ of the scalp. 


\section{CASE REPORT}

A 52-year-old white male patient reported a hyperchromic spot on the scalp that had been present since the age of 2 years. At age 20, patient developed an alteration on lesion surface which presented an irregular central elevation. At that time, a biopsy of that site was performed, and the histopathological report concluded as a benign pigmented lesion (sic).

The lesion continued a slow and progressive growth over the years. Medical assistance was sought due to recent increase in size and emergence of nodules and ulcerated areas.

Physical examination showed a grayish, $10-\mathrm{cm}$ tumor in the left occipital region with superimposed erythematous to violaceous nodules and crusting (Figure 1). An incisional biopsy was performed, with histopathological report of superficial and deep, predominantly dendritic, BN (Tièche-Jadassohn nevus, or common BN) (Figure 2). However, although no signs of malignancy were observed in the specimens studied, additional biopsies were suggested in other areas of the neoplasia to guarantee the representativity of the lesion, due to the clinical-histopathological disparity. Three new speci- mens revealed a malignant melanoma, Clark level IV, $7.5 \mathrm{~mm}$ thick (Breslow), occupying until the deep base of sections (Figure 3).

Patient was referred to the head and neck surgery unit and underwent complete excision of the neoplasia, but died shortly after.

\section{DISCUSSION}

$\mathrm{BN}$ occurs more frequently in children and young adults, but may be observed at any age, including including congenital presentation as in the case reported. It is usually a small lesion, with predilection for the buttocks, scalp and back of hands and feet. ${ }^{4,8,9}$

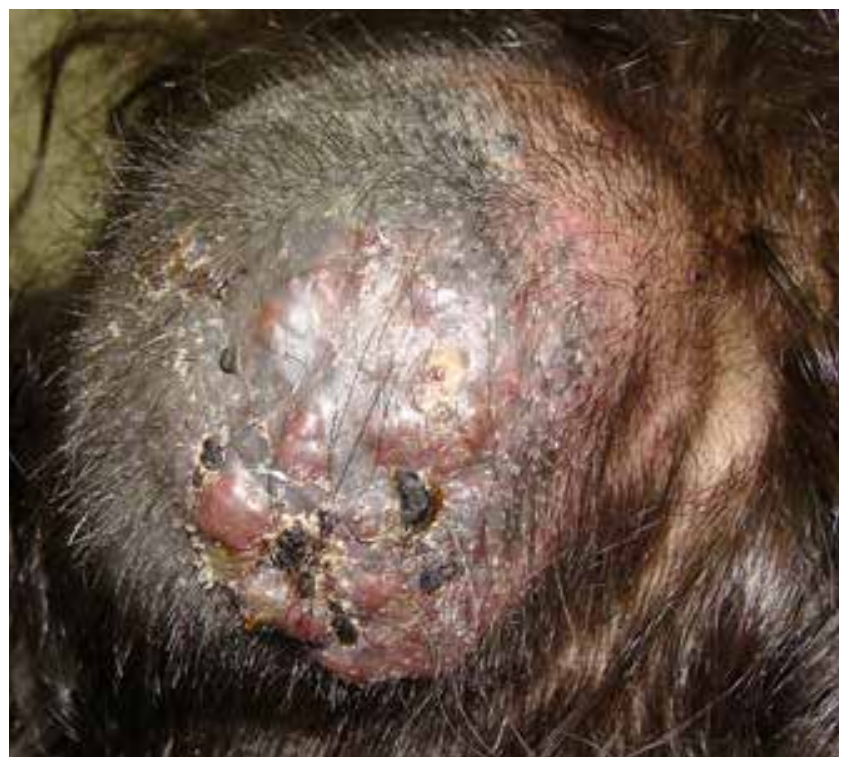

Figure 1: Melanoma over blue nevus. Tumoral lesion in the left occipital region, of greyish coloration in the background, exhibiting erythemato-violaceous nodules and areas of ulceration covered by hematic crusts. The greyish and homogeneous portion to the left is probably representative of the preexistent blue nevus, whereas the superelevation to the right characterizes the melanoma

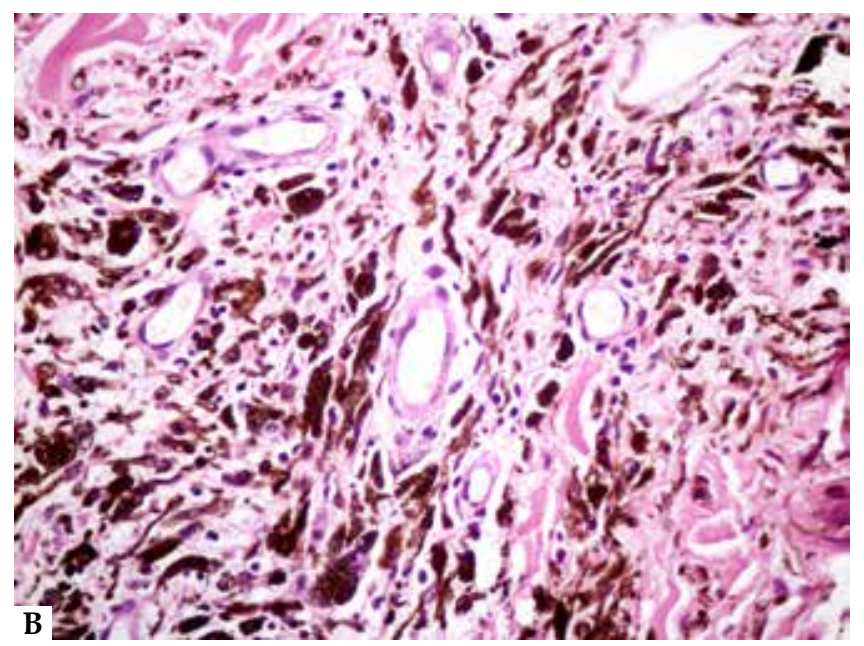

Figure 2: A. Dendritic blue nevus

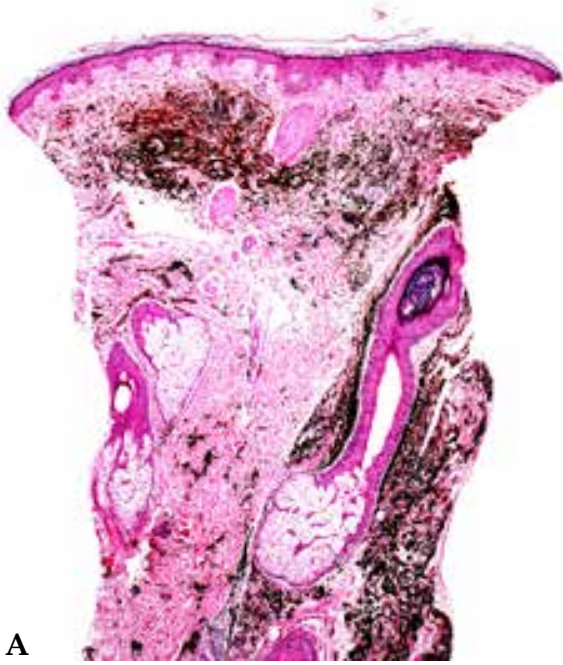

of Tièche-Jadassohn. Proliferation of melanocytes predominantly dendritic, intensely pigmented, occupying the papillary and reticular dermis, positioned around cutaneous annexes (HE, 40x); B. Dendritic blue nevus of Tièche-Jadassohn. Detail of dendritic melanocytes arranged in the reticular dermis among the collagen bundles (HE, 400x)

Conversely, the giant congenital $\mathrm{BN}$ is a large and single lesion, affecting the whole scalp in most cases. ${ }^{9}$

The authors disagree about the histopathological classification of $\mathrm{BN}$, but there is consensus concerning the subtypes: common, cellular (neuronevus of Masson), epithelioid and combined. Also, the opinion that the onset of melanoma over $\mathrm{BN}$ is more frequently observed in lesions on the scalp and more often associated with the cellular histological subtype is unanimous, even though there are reports, such as the present one, of melanomas arising over common BN. ${ }^{4,6,9}$

Besides the histological types mentioned above, there is, in the literature, the description of the malignant BN. But, like other authors, we consider this term inappropriate and we prefer referring to these cases as a subtype of melanoma, which, as opposing to the classic one, arises in the dermis over previous BN lesions. ${ }^{5,8}$

Two important factors weight over the therapeutic choices for congenital nevi: the layer of skin where the melanoma usually arises and the age group most frequently affected. It is known that 

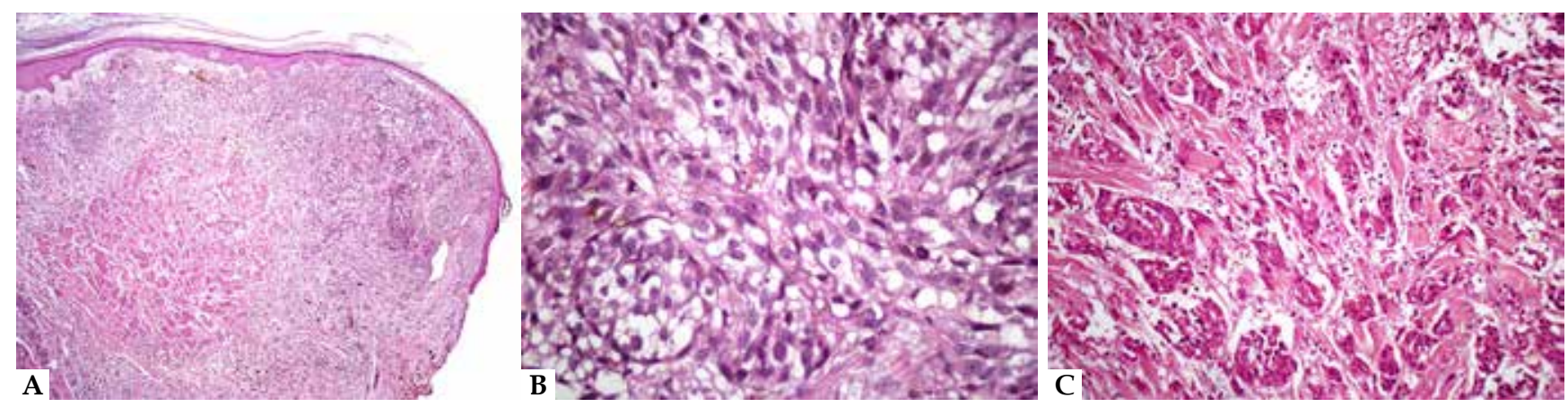

Figure 3: A. Malignant melanoma. Sheet of atypical melanocytes throughout papillary and reticular dermis (HE, 40x); B. Malignant melanoma. Pleomorphic melanocytes arranged in confluent nests. Mitotic figures are present (HE, 400x); C. Malignant melanoma. Necrotic melanocytic nests in the reticular dermis (HE, 100x)

more than $2 / 3$ of melanomas arising from congenital giant nevi develop from melanocytes located in the dermis, as opposed to the melanomas originating from small and medium nevi, which usually have an epidermic origin. ${ }^{1}$ For this reason, early diagnosis of melanoma in small and medium nevi may be easier than in giant congenital nevi. When melanoma occurs over a giant congenital nevus, the diagnosis commonly happens in more advanced phases of the neoplasia, due to nodules or ulceration, and there may be metastases at the moment of diagnosis. ${ }^{2}$ Another interesting fact, which influences the treatment, is that development of melanomas in giant nevi usually occurs before puberty, in the first five years of age; as for the small and medium nevi, it routinely occurs after puberty. ${ }^{2,3,8}$ Here we report a patient whose melanoma, which arose over a medium congenital BN, was diagnosed at 52 , in an advanced stage. Therefore, in the reported case, the link between the size of nevus and the age of onset of the melanoma conforms the data from literature.

For these reasons, surgical resection of giant congenital nevi is frequently recommended before puberty and when located in areas difficult to monitor. ${ }^{9,10}$ Notwithstanding, complete excision is many times difficult, or even impossible, due to its size and multiple satellite lesions. ${ }^{1}$ As to the small and medium nevi, prophylactic excision is not essential, because clinical observation is considered capable of detecting signs of malignization. Once removal of these nevi is decides, it should be carried out before puberty. ${ }^{1} \square$

\section{REFERENCES}

1. Paschoal FM. Nevo melanocítico congênito. An Bras Dermatol. 2002;77:649-56.

2. Price HN, Schaffer JV. Congenital melanocytic nevi - when to worry and how to treat: Facts and controversies. Clin Dermatol. 2010;28:293-302.

3. Yun SJ, Kwon OS, Han JH, Kweon SS, Lee MW, Lee DY, et al. Clinical characteristics and risk of melanoma development from giant congenital melanocytic naevi in Korea. Br J Dermatol. 2012;166:115-23.

4. Khanna A, Burra U, Saxena S, Bhandari. Infiltrating giant congenital cellular blue nevus of neck presenting as melanoma. Int J Pathol. 2005;4:1-5.

5. Hu W, Nelson JE, Mohney CA, Willen MD. Malignant melanoma in pregnant african american woman. Dermatol Surg. 2004;30:1530-2.

6. Khashashneh I, Shatnawi M, Tawalbeh A, Alhaji M, Rajjal M. Giant congenital hairy nevus on the scalp. Sudan J Med Sci. 2008;3:343-7.

7. Purdue MP, From L, Armstrong BK, Kricker A, Gallagher RP, McLaughlin JR, et al. Etiologic and Other Factors Predicting Nevus-Associated Cutaneous Malignant Melanoma. Cancer Epidemiol Biomarkers Prev. 2005;14:2015-22.

8. Zembowicz A, Phadke PA. Blue nevi and varients an update. Arch Pathol Lab Med. 2011;135:327-36.

9. Nakamura $Y$, Shibata-Ito M, Nakamura $Y$, Ishitsuka $Y$, Fujisawa $Y$, Kawachi $Y$, et al: Malignant Blue nevus arising in a giant congenital cellular blue nevus in an infant. Pediatr Dermatol. 2012;29:651-5.

10. Aloi F, Pich A, Pippione M. Malignant cellular blue nevus: a clinicopathological study of 6 cases. Dermatology. 1996;192:36-40.

How to cite this article: Ribeiro CS, Serpa SS, Jeunon-Sousa MA, Jeunon T. Melanoma arising in a congenital intermediate common blue nevus of the scalp - Case report. An Bras Dermatol. 2016;91(4):514-6. 